Published online 2017 April 13.

Abstract

\title{
Comparison of Low Dose and Standard Dose Abdominal CT Scan in Body Stuffers
}

\author{
Zahra Mahboubifooladi, ${ }^{1, *}$ and Hooman Bahramimotlagh ${ }^{1}$ \\ ${ }^{1}$ Shahid Beheshti University of Medical Sciences, Tehran, Iran \\ "Corresponding author: Zahra Mahboubifooladi, Shahid Beheshti University of Medical Sciences, Tehran, Iran. E-mail: mahboubiz@yahoo.com \\ Received 2016 December 21; Accepted 2017 February 08.
}

\begin{abstract}
Background: Body stuffer determination is an important concern in emergency departments for referred patients with suspicion of swallowing drug packets. Because of small packet size, plain radiography is of little value in most patients. On the other hand, abdominal and pelvic CT scan is burdened by high cost and radiation dose. This study was performed to evaluate the sensitivity of low-dose CT scan in comparison with conventional one. So, the former can be used as an alternative screening and follow up tool for suspected body stuffers

Methods: In this prospective study, suspected body stuffers who were referred to radiology department, underwent two different protocols of abdominal and pelvic non-contrast CT scan: low-dose (equivalent dose of conventional abdominal x-ray) and standard dose. Standard dose CT scan was considered as the gold standard and low-dose protocol was compared with that for image quality and packet detection rate.

Results: The study consisted of 40 patients (all men, with mean age of $33.38 \pm 7.4$ years). CT evaluation with standard dose were positive in 22 patients (55\%). Only 1 (2.5\%) CT scan assigned as unacceptable by each radiologist in low-dose group. Noise average of low-dose protocol, was approximately 7 times greater than standard dose group, while DLP and ED are 9.7 times smaller. In comparison with standard dose CT scan, low-dose group had a sensitivity of $86.46 \%$, specificity of $100 \%$, PPV and NPV of $100 \%$ and $85.71 \%$. Discussion: Accuracy of low-dose CT scan for detection of baggies larger than $1 \mathrm{~cm}$, approaches 100\%. Therefore, it looks an appropriate screening method for body stuffers, especially when the packages are larger.
\end{abstract}

This is an abstract presented in the 33rd Iranian congress of radiology (ICR) and the 15th congress of Iranian radiographic science association (IRSA) 